\title{
La inclusividad del alumnado con deficiencia auditiva
}

Natalia Chust Company

al239442@alumail.uji.es

Clara Hernández Peiró

al239444@alumail.uji.es

Laura Luján Parra

al239279@alumail.uji.es 
I. Resumen

La educación inclusiva es un proceso de abordar y responder a la diversidad de necesidades de todos los alumnos a través de prácticas inclusivas en el aprendizaje, las culturas y las comunidades y reducir la exclusión dentro de la educación. Implica cambios y modificaciones en el contenido, los enfoques, las estructuras y las estrategias, con una visión común que cubra a todos los niños del rango apropiado de edad y una convicción de que es la responsabilidad del sistema ordinario educar a todos los niños. (UNESCO, 2006)

Bajo esta perspectiva, se entiende que todo el alumnado debe recibir una educación integral, que atienda a sus necesidades de desarrollo y le brinde una igualdad de oportunidades.

La deficiencia auditiva es un déficit sensorial en la percepción de las cualidades del sonido. El término hipoacusia hace referencia a una pérdida parcial en la percepción auditiva, mientras que sordera se relaciona con la pérdida total o casi total de la audición.

Tras estas consideraciones generales se ha elaborado el proyecto de innovación curricular Chust, Hernández, Luján (2012): La inclusividad del alumnado con deficiencia auditiva.

Con el objetivo de esclarecer el nivel de inclusividad existente en la educación del alumnado con deficiencia auditiva se administró un cuestionario de 12 ítems a 20 profesores pertenecientes a un centro ordinario de atención preferente al alumnado sordo. Una vez analizados los resultados obtenidos se observó la necesidad de atender de una manera más inclusiva a los discentes.

El proyecto curricular que aquí se presenta, pretende atender a la diversidad desde una perspectiva innovadora que permita al alumnado con un hándicap comunicativo, sordera o hipoacusia beneficiarse directa o indirectamente de una educación que les ofrezca las mismas posibilidades que a sus pares en la formación integral de su persona.

Palabras clave: inclusividad, deficiencia auditiva, innovación, comunidad educativa.

\section{Introducción}

La Integración escolar llegó a los centros educativos españoles a mediados de los 80. La filosofía de este movimiento, que supuso un verdadero cambio en el sistema educativo, se centra básicamente en la obligación de este en atender las Necesidades Educativas Especiales de los educandos con Dificultades de Aprendizaje dentro del marco escolar ordinario.

En los años 90, surgen, a partir de los Movimientos Integradores de educación, los planteamientos de Inclusividad Educativa. Será obligación del Sistema Educativo responder a las Necesidades Educativas puntuales 
o permanentes de todos los discentes; atender las individualidades de todo el alumnado en el proceso de enseñanza/ aprendizaje dentro del marco educativo ordinario regido por los valores de equidad e igualdad, y no únicamente de aquellos alumnos que presentan Necesidades Educativas Especiales.

La Ley Orgánica, 2/2006 del 3 de mayo, de Educación, la cual rige el Sistema Educativo español actual, se centra en los principios anteriormente citados de Educación Inclusiva. La misma, expone en el Título segundo, equidad en educación, la obligación del Sistema Educativo de otorgar los recursos necesarios para atender al alumnado con Necesidad Específica de Apoyo Educativo, Altas Capacidades o Incorporación Tardía al Sistema Educativo, siempre que sea posible dentro del marco de educación ordinaria.

Sin embargo, a pesar de que la atención al alumnado con Necesidades Educativas Especiales en el Sistema ordinario de Educación es real, deja de ser equitativa respecto del alumnado sin Dificultades de Aprendizaje. Normalmente, la atención a los discentes con Necesidades Educativas Especiales se lleva a cabo por especialistas fuera del aula ordinaria de referencia para estos alumnos. Una medida de atención a la diversidad, que aunque sumamente necesaria e integradora, deja de lado los principios de una Escuela Inclusiva totalmente en contra de la segregación del alumnado.

Por ello, se pretende con el proyecto de innovación curricular que se ha diseñado atender a la diversidad desde una perspectiva innovadora que permita a los discentes con sordera o hipoacusia beneficiarse directa o indirectamente de una educación que les ofrezca las mismas posibilidades que al resto del alumnado en la formación integral de su persona.

Se hará una breve alusión conceptual de los términos hipoacusia y sordera:

Se entiende por hipoacusia una pérdida parcial en la percepción auditiva, mientras que sordera se relaciona con la pérdida total o casi total de la audición.

En función a la zona del oído en el que se encuentra la lesión, el grado de pérdida auditiva y el momento en el que se sufrió la pérdida se puede realizar diferentes clasificaciones:

- Según la zona del oído afectada (externa, media, y/o interna) se encuentra: sorderas conductivas o de trasmisión (afectación del oído externo y/o medio), neurosensoriales o de percepción (disfunción del oído interno y/o nervio auditivo) o mixtas.

- Según el grado de la pérdida se puede clasificar: deficiencia auditiva leve y ligera (umbral del audición entre 0 y $40 \mathrm{~dB}$ ), deficiencia auditiva media o moderada (umbral de audición entre 40 y $70 \mathrm{~dB}$ el cual hace referencia a la intensidad de una conversación normal), deficiencia auditiva severa 
(umbral de audición entre 70 y $90 \mathrm{~dB}$ ) y deficiencia auditiva profunda (umbral de audición por encima de $90 \mathrm{~dB}$ ). La pérdida total de la audición denominada cofosis supondría restos auditivos por encima de los $120 \mathrm{~dB}$.

- Según en el momento en el que se sufrió la pérdida: prelingüística o prelocutiva (cuando la pérdida se produce antes de que se haya completado las etapas iniciales del desarrollo del lenguaje oral) y postlingüística o poslocutiva (posterior a la primera infancia y con un grado de repercusión menor sobre el desarrollo).

El colectivo sordo en los centros escolares es considerado un grupo de necesidades educativas especiales al cuál se le debe brindar recursos escolares específicos como maestro/a de audición y lenguaje y maestro/a de pedagogía terapéutica. Dicha concepción es necesaria y real en el ámbito educativo, pero insuficiente. Es preciso dar respuesta a las necesidades específicas del desarrollo educativo de este colectivo, pero a su vez, es imprescindible una actuación paralela en otras áreas del desarrollo, así como la actuación en otros contextos diferentes al educativo, como el familiar. Sólo de este modo se realizará el progreso de una escuela integradora a una escuela inclusiva. $Y$ este éste el objetivo general del programa de innovación curricular que se ha llevado a cabo.

A priori de la elaboración del proyecto de innovación curricular que se presenta, se reflexionó sobre las posibles necesidades de la escuela respecto al tratamiento inclusivo del alumnado con deficiencia auditiva en las aulas. Para contextualizar y determinar estas necesidades reales se suministró un cuestionario de doce ítems a veinte de los docentes de un CEIP (Centro Educativo de Infantil y Primaria) de Integración Preferente de alumnado con deficiencia auditiva de la localidad de Valencia.

Se distribuyó entre una muestra variada del profesorado (en cuanto a edad, años de experiencia docente y nivel educativo impartido) un cuestionario de preguntas abiertas donde se cuestionaba los aspectos a mejorar en el centro respecto al tratamiento de los discentes con eficiencia auditiva, las posibilidades de mejora, la valoración que individualmente se hace en las aulas de la diversidad, la necesidad actual de medidas para fomentarla, así como la forma en que cada uno de ellos la trabaja en el aula. También se cuestiona a los docentes sobre su conocimiento respecto a la importancia del lenguaje como elemento básico de acceso al currículum, de la existencia de programas específicos para su desarrollo en el centro y del conocimiento por su parte de este tipo de programas. Se preguntó, también, a los docentes sobre la formación y los recursos que disponían para atender de forma óptima al alumnado con deficiencia auditiva.

Mediante el análisis de estos cuestionarios se observó que todos los docentes consideraban que es necesario realizar mejoras en el funcionamiento del centro en aspectos muy variados. Los aspectos más destacados por los docentes como deficitarios en el centro fueron la atención individualizada del alumnado con deficiencia auditiva dentro de su aula ordinaria, la coordinación entre los docente, y entre estos y el 
equipo directivo, la mejora de las instalaciones y los recursos, así como una mayor participación y coordinación con las familias del alumnado.

Por otro lado, a pesar de que todos los docentes contestaron que consideran positivo valorar la diversidad en las aulas, las respuestas a los cuestionarios denotaron que no existe una coordinación que permita su tratamiento. La atención a la diversidad se reduce a las medidas que rige la legislación, es decir, atención individualizada por parte de los maestros especialistas de audición y lenguaje y pedagogía terapéutica, y en la elaboración de las ACIS.

Respecto a las pregunta acerca del lenguaje como elemento de acceso al currículum, los docentes señalaron que un buen desarrollo del lenguaje es un requisito necesario para el dominio de los aprendizajes escolares, pero que el centro, a pesar de contar con una amplia población de alumnado sordo, no cuenta con programas específicos para su desarrollo. Además, la mayor parte de los docentes, desconocen programas que potencien su adquisición.

Así mismo, la gran mayoría considera que el alumno sordo tiene más posibilidades de tener problemas sociales o emocionales. La principal causa de estas dificultades señalada por los docentes son las dificultades con el lenguaje y sus problemas de autoestima e inseguridad, que complican las relaciones con los demás.

En cuanto a posibilidades y oportunidades para el desarrollo integral de los discentes con deficiencia auditiva, sus docentes también coinciden en indicar que hay una diferencia con el alumnado que no presenta un hándicap auditivo. Entre los motivo más señalados están la falta de recursos tanto materiales como personales en los centros educativos, que permitan ofrecer a este alumnado una educación acorde con sus necesidades, así como una falta de flexibilidad en la sociedad, señalando posibles limitaciones en las relaciones sociales y en el acceso laboral, debido fundamentalmente a sus dificultades comunicativas y la falta de recursos que permitan su superación.

Por este motivo, se decide iniciar la elaboración del proyecto de innovación curricular que se presenta para satisfacer las necesidades investigadas en un contexto de atención preferente de alumnado con deficiencia auditiva.

\section{Objetivos}

\section{Objetivo general}

- Potenciar la inclusividad del alumnado sordo a través de la formación a docentes, familias y discentes.

\section{Objetivos específicos}

- Potenciar varios canales comunicativos de transmisión de la comunicación. 
- Introducir los conceptos básicos de las Unidades Didácticas mediante dos inputs sensoriales (visual y auditivo).

- Formar al docente en pautas comunicativas y metodológicas qué favorezcan el tratamiento inclusivo (con las mismas posibilidades) del alumnado con deficiencia auditiva al aula.

- Formar al docente en el conocimiento del Bimodal y del signos básicos de la Lengua de Signos para introducirlos en la lengua vehicular de las sesiones que configuran las Unidades Didácticas de la Programación de Aula.

- Posibilitar un mejor aprendizaje del lenguaje oral a través de las ayudas viso-gestuales que proporcionan los signos.

- Hacer consciente al alumnado sin ningún handicap sensorial de la necesidad de tener en cuenta unas pautas para comunicarse con el alumnado con deficiencia auditiva.

\section{Material y método}

\section{Destinatarios}

Agentes educativos a los que va dirigido el proyecto de innovación educativa.

\subsection{Alumnos con deficiencia auditiva}

Al potenciar el canal visual como medio vehicular de las conversaciones mediante el Bimodal y haciendo uso por parte de todos los miembros del grupo/clase de unas pautas comunicativas que ayudan al alumno/a con deficiencia auditiva a participar y entender los mensajes comunicativos del aula; al utilizar la creatividad como medio de expresión de sus emociones, sentimientos y sensaciones y al mejorar la inteligencia emocional de este alumnado, se está intentando que todo el alumnado tenga las mismas posibilidades comunicativas dentro del grupo/clase independientemente de si tiene o no un hándicap sensorial.

El proyecto de innovación educativa va dirigido tanto a los alumnos sordos de padres sordos qué emplean un canal comunicativo gestual, como a los alumnos sordos de padres oyentes.

Si el alumno o los alumnos con deficiencia auditiva pertenecen a la Comunidad Sorda y el canal vehicular de comunicación materna es la Lengua de Signos, este modelo favorecerá qué el alumnado recientemente incorporado al Sistema Educativo no perciba una gran distancia entre la modalidad comunicativa oral y la gestual.

Empleando el Bimodal como un sistema de refuerzo a la lengua oral se intenta no sobrecargar la atención del alumno con deficiencia auditiva hacia los mensajes orales.

$\mathrm{Si}$ el alumno realiza un sobreesfuerzo atencional su memoria de trabajo y capacidad perceptiva hacia los estímulos disminuye. 
Se debe tener presente que, a pesar de que los alumnos con un hándicap auditivo empleen prótesis, estas no conseguirán "eliminar" la sordera, únicamente incrementar su funcionalidad auditiva. Por este motivo, es sumamente importante el refuerzo del lenguaje oral con estímulos visuales.

\subsection{Resto del alumnado de la clase sin ningún hándicap físico, psíquico o motriz}

Con la utilización del Bimodal, no únicamente serán beneficiarios de la puesta en marcha del proyecto de innovación curricular presentado el alumnado con deficiencia auditiva sino también el resto de alumnado del grupo/clase. $Y$ es que, al presentar y transmitir un mismo concepto mediante dos inputs sensoriales (el auditivo y visual), y con la escritura, serán tres entradas sensoriales (auditiva, gestual y gráfica) las qué potenciarán la interiorización de los contenidos conceptuales del currículo escolar. Una metodología que ayudará también a aquellos alumnos con una capacidad inferior de retención.

\subsection{Docentes}

Con este programa se pretende capacitar a los docentes de conocimientos del lenguaje Bimodal, creatividad e inteligencia emocional. Esta formación aportará al docente aprendizajes aplicables a la gestión de la vida privada.

Además puede la aplicación de este programa suponer que se establezca unas relaciones más estrechas entre los maestros/as tutores y los docentes especialistas y el fomento del trabajo cooperativo y en equipo.

\subsection{Familia}

Uno de los objetivos de este proyecto es ayudar a los padres en la relación con sus hijos/as, proporcionarles diferentes recursos para fomentar la comunicación con ellos/as, y comprender las repercusiones que conlleva el déficit auditivo en el desarrollo.

Los padres, en etapas tempranas del desarrollo del niño, son los principales agentes estimuladores, acondicionadores y formadores, por lo que es importante que tengan en consideración cuáles son las conductas esperables en sus hijos/as y las formas de intervención efectivas para ellos.

Los trastornos en el desarrollo del lenguaje colocan a los niños en riesgo de presentar dificultades sociales, emocionales y académicas en el largo plazo. Mediante este programa se pretende desarrollar intervenciones implementadas por los padres, donde éstos se convierten en los agentes primarios de intervención, y aprenden cómo facilitar el desarrollo del lenguaje en contextos cotidianos y naturales. 


\section{Descripción del proyecto}

El proyecto de innovación curricular presentado se divide en tres focos de actuación bien diferenciados.

Por un lado, nos encontramos con la atención hacia la organización docente, en donde se desarrolla contenidos relacionados con la comunicación con los alumnos (técnicas y tipos de lenguajes aumentativos de comunicación) para favorecer su inclusión dentro de las aulas ordinarias.

Por otro lado, nos encontramos con la intervención directa en el aula con el alumnado, en donde se trabajarán la creatividad y la educación emocional de estos alumnos.

Además también existe un ámbito de intervención referido al ámbito familiar, en el cuál se pretende ampliar el conocimiento de los padres alrededor de la deficiencia auditiva y dotarles de técnicas específicas para fomentar el lenguaje.

En definitiva nuestro proyecto se divide en tres ramas diferentes, las cuáles se llevarán a cabo de forma independiente, pero dentro del mismo periodo temporal. Es decir, la temporalización es conjunta, pero el desarrollo de cada bloque es independiente. En este sentido, la atención referida a los alumnos se realizará en horario de tutorías. La atención a los docentes en las horas complementarias de la jornada laboral y, finalmente, la atención a los padres, en horario extraescolar. Todo ello se llevará a cabo a lo largo de un curso escolar.

\section{1 Ámbito docente}

Pautas para el proceso comunicativo con el alumnado sordo.

En primer lugar, se orientará al docente hacia pautas metodológicas para favorecer la comunicación con el alumnado con deficiencia auditiva.

Por un lado, se proporcionará al docente de alumnos/as con deficiencia auditiva recomendaciones sobre las pautas comunicativas que tiene que tener en cuenta para abolir las posibles barreras que dificultan al alumno con deficiencia auditiva seguir las sesiones y los diálogos que se desarrollan en el aula.

Estas pautas comunicativas harán referencia a técnicas efectivas para llamar la atención del discente con deficiencia auditiva, estrategias a emplear durante la conversación que favorecen la comprensión del discente sordo/a, y recomendaciones sobre el uso del espacio y la distribución óptima del aula para que el alumno/a con deficiencia auditiva no pierda mensajes comunicativos que se den dentro del desarrollo normal de las sesiones de enseñanza/aprendizaje.

El docente, también tendrá que formar al resto del alumnado sin hándicap auditivo en estas pautas comunicativas para favorecer la integración del alumno/a con deficiencia auditiva.

Por otro lado, se proporcionará recursos, materiales y sesiones formativas para establecer un cambio metodológico en el canal vehicular de comunicación al aula de Educación Infantil. 
Fundamentalmente el canal primordial comunicativo al ámbito escolar es el oral. Si únicamente se potencia este canal, el alumno sordo tiene que efectuar un sobreesfuerzo constante por mantener la atención a las explicaciones del docente y a las intervenciones de los compañeros. Este sobreesfuerzo atencional puede repercutir en una carencia en la capacidad perceptiva y de memoria de trabajo, y ser causa de un bloqueo en las capacidades cognoscitivas de procesamiento de la información.

Con el fin de que el alumno/a con deficiencia auditiva reciba un tratamiento inclusivo de su hándicap en el aula será imprescindible que se potencien otros canales comunicativos de transmisión de la información, además del oral.

No únicamente será necesario potenciar vías comunicativas alternativas en el canal oral para que el alumno pueda percibir sin sobreesfuerzo los contenidos curriculares que se desarrollan en el aula. Si no también porque las posibles carencias atencionales del sujeto se apoyen mediante un canal visual que le permita ser capaz de desarrollar una capacidad lingüística para adquirir un código lingüístico. De esta manera será posible establecer relaciones sociales, conocer el entorno y estructurar su propio pensamiento.

Por lo tanto, la finalidad primordial de este proyecto es formar al docente en unas pautas comunicativas que fomentan el canal comunicativo visual. Se pretende que el docente adapte su metodología y fomente las relaciones comunicativas a través de un sistema alternativo de comunicación: el Bimodal.

La comunicación en Bimodal consiste en la utilización simultánea del habla y los signos. Es decir es una comunicación oral-auditiva y visualgestual. El Bimodal pretende "casar" cada palabra del lenguaje oral con gestos de la Lengua de Signos pero siguiendo la estructura sintácticagramatical del lenguaje oral.

La comunicación Bimodal puede ajustarse a la lengua hablada en diferentes grados, según los objetivos que se pretenda conseguir.

Empleando el Bimodal en el aula, es decir, realizando signos gestuales de la Lengua de Signos de forma simultánea al habla, se pretende fomentar el canal visual como medio comunicativo sin dejar de lado las producciones orales.

No se pretende que se lleve a cabo una comunicación Bimodal pura, si no que se signe únicamente aquellos signos que representan los conceptos más básicos de la comunicación.

A medida que los canales de entrada de información son mayores, el input que recibe el alumno también será mayor. Por este motivo, emplear el Bimodal, no únicamente favorecerá al alumno con deficiencia auditiva sino al resto del alumnado sin ningún hándicap sensorial.

Además, el hecho de que los alumnos aprendan a signar repercutirá en una mejora en el desarrollo de la motricidad fina (objetivo qué forma parte de las programaciones de Primer curso de Educación Infantil).

Las sesiones formativas en Bimodal que se dirigen al docente y el uso del mismo en la comunicación ordinaria del aula contribuirán a eliminar al 
máximo la distancia entre el ámbito familiar y escolar de los alumnos con deficiencia auditiva de padres deficientes auditivos qué emplean el Lenguaje de Signos como sistema lingüístico de comunicación habitual.

Los alumnos deficientes auditivos de Educación Infantil recientemente incorporados al Sistema Educativo y que están acostumbrados a que la lengua vehicular materna sea el Lenguaje de Signos, experimentan un gran desconcierto cuando al ámbito escolar únicamente se utiliza el lenguaje oral como vía de comunicación.

\section{2 Ámbito del alumnado}

Programa basado en la creatividad como medio de expresión.

Con este programa se pretende estimular la creatividad en cada alumno o alumna favoreciendo también su ajuste socio-emocional. Mediante éste se pretende que los niños sean capaces de ser seres creativos libres para que el ingenio les posibilite crecer en aprendizajes, puesto que en una clase no sabemos cuanta creatividad se desperdicia por enfatizar el aprendizaje. Se considera interesante utilizar algunas de las actividades de este programa que ayuden a desarrollar el pensamiento creativo en los alumnos. Estas actividades creativas ayudan a reconocer y a celebrar el aspecto único y la diversidad de los niños así como también ofrecer oportunidades excelentes para individualizar sus actos y expresar lo que sienten en cada momento.

Por tanto con éste se pretende, fundamentalmente, que los niños sean capaces de mejorar el control de sus emociones y crezcan y mejoren sus habilidades sociales. Para que todo esto sea posible se utilizarán diferentes formas y técnicas de expresión como son: el dibujo, la acuarela, las ceras y la arcilla, entre otros materiales. En estos procesos de creación es el alumno el que marca sus pautas creativas y el desarrollo de la idea, seleccionando su forma de expresión y percepción visual.

Por último añadir que con este programa no se pretende trabajar la creatividad de forma paralela al resto de áreas curriculares, sino que se trabaje esta capacidad y a su vez, se mejoren otros contenidos curriculares pertenecientes a áreas como matemáticas, lenguaje, etc.

\section{3 Ámbito familiar}

Potenciar la comunicación con el alumnado sordo en el ámbito familiar. El objetivo principal y único de dicho apartado es lograr desarrollar en los padres una conciencia sobre el déficit auditivo. Por un lado se persigue el conocimiento del déficit en sí, y por otro sus repercusiones dentro de las diferentes áreas de desarrollo de aquellos alumnos que lo padezcan. Además también se espera fomentar en este aparatado que los padres aprendan diferentes técnicas que puedan potenciar la comunicación con sus hijos, ya que en numerosas ocasiones se encuentran faltos de recursos, quedando este contexto (el eje de referencia más significativo para todo niño) fuera del proceso de intervención. 
La razón por la cual se desarrolla dicho apartado y se incluye dentro de este proyecto de innovación es principalmente ese. La familia es el contexto más importante en el desarrollo de cada individuo. En ella se encuentran las figuras que más influyen en su desarrollo, por tanto, es preciso formar a dichas personas con el objetivo de fomentar un mejor desarrollo atendiendo de forma más específicas a las necesidades especiales de este colectivo.

Para poder conseguir de forma eficiente todos los objetivos que se pretenden en este apartado se realizarán diferentes sesiones con los padres. Cada una de las sesiones trabajará un objetivo específico, de forma que se pueda ir avanzando en los conocimientos de forma gradual.

Se seguirá las siguientes pautas metodológicas:

- Partiremos de las necesidades de los padres y de los niños para establecer los contenidos.

- Se utilizaran unos materiales lo más lúdicos y aclaradores posibles.

- Las sesiones se harán en grupo, ofreciendo la posibilidad para aquel que lo desee de sesiones individualizadas para concretar las pautas más específicas de cada niño.

- Se trabaja un objetivo por sesión para que los padres que acudan al programa entiendan mejor todo lo que se les explica con coherencia. En este sentido se partirá de temas más generales y poco a poco se irán incluyendo otros más específicos.

Por último destacar que las sesiones tendrán una estructura tipo taller. Las primeras tendrán un carácter más teórico, ya que abordan temas de entendimiento necesarios para poder pasar a las aplicaciones prácticas. Podríamos decir que constituyen la base para que las posteriores aplicaciones prácticas tengan sentido. El resto de las sesiones ya tendrán un enfoque mucho más práctico, las cuales partirán de la base teórica inicialmente trabajada.

Aún así es pertinente señalar que el volumen de sesiones teóricas es bastante inferior a las prácticas ya que lo que se persigue no es desarrollar un conocimiento teórico, sino un conocimiento práctico que se pueda utilizar eficientemente.

\section{Resultados}

El proyecto de innovación presentado entiende la educación del alumnado, desde la perspectiva de Inclusión Educativa, como un proceso de formación íntegro de los educandos. Se atiende al alumnado con deficiencia auditiva en función de sus necesidades; necesidades que implican a los diferentes agentes educativos y entornos donde el alumno se desenvuelve.

La formación plena del alumnado en los diferentes ámbitos que integran su desarrollo personal, exige la necesidad de coordinación entre los diferentes agentes educativos, lo que requiere un esfuerzo por parte 
de todos ellos para la consecución de los objetivos del programa propuesto. Así, se precisa de una gran implicación del profesorado para su formación en el lenguaje bimodal, creatividad, habilidades sociales, y asesoramiento de procesos comunicativos óptimos a familias, con el fin de ayudar a alcanzar una educación de calidad, entendida ésta como una educación que ofrece al alumnado posibilidades reales de alcanzar su máximo desarrollo integral.

Respecto a las limitaciones determinadas en este proyecto de innovación educativa, cabe hacer referencia a la aún existente tradición instruccional de segmentación del conocimiento académico. Desde la creación de la escuela hasta la actualidad, la parcelación de la educación formal en áreas curriculares impermeables genera dificultades en la aplicación de las diferentes áreas que integran y componen el proyecto de innovación curricular presentado. En ocasiones, la excesiva segmentación de la educación formal en áreas de conocimiento concretas puede dejar de lado la formación en competencias educativas como las habilidades sociales o creatividad.

Por otro lado se hará mención también, a que la formación de los docentes, la familia, así como del alumnado, tanto con hándicap auditivo como sin deficiencias sensoriales, supone un primer paso para facilitar la participación del alumno/a con deficiencia auditiva en su entorno, si bien, para una plena inclusión en él, sería necesario llevar a cabo diferentes estrategias de sensibilización social a través de la coordinación con otros servicios de la comunidad, es decir, no basta con la formación de aquellos agentes educativos directamente implicados en el desarrollo del alumno, sino del entorno que, de una forma indirecta, también interviene en su formación, pues cabe destacar que hay una responsabilidad social colectiva en relación con la educación.

Con este proyecto de innovación curricular diseñado se ha pretendido responder a unas carencias reales y evidentes de escuelas que mayoritariamente atienden a la formación de discentes con deficiencias auditivas. La puesta en práctica del mismo podría ser el inicio de dar respuesta de forma inclusiva a las necesidades específicas del alumnado con deficiencia auditiva.

\section{Discusión y conclusiones}

Este proyecto de innovación ha conseguido que la educación del alumnado con déficit auditivo sea vista desde una perspectiva de inclusión donde se le dote de las mismas posibilidades y oportunidades que al resto ya que, entre otras cosas, el mismo dota de recursos materiales y personales a los centros educativos haciendo posible que todo el alumnado reciba una educación acorde a sus necesidades. Por tanto, se puede afirmar que El Proyecto de Inclusión del Alumno Sordo atiende a la diversidad desde una perspectiva innovadora que ofrece a todo el alumnado beneficiarse directa o indirectamente de una educación con las mismas posibilidades en la formación integral de su persona. 
VII. Bibliografía

CORBALÁN, F. J. (2003): CREA: inteligencia creativa: una media cognitiva de la creatividad, Ediciones TEA, Madrid.

DE Bono, E. (2008): Creatividad: 62 ejercicios para desarrollar la mente, Ediciones Paidós, Barcelona.

ForTeS Del VAlLE, M.C. (2000): Bases psicológicas de la educación especial, Promolibro, Valencia.

Gallego Ortega, J. L. (1999): Calidad en la intervención logopédica, Ediciones Aljibe, Málaga.

Jiménez ToRres, M.G. y M. LóPez SÁnCHez (2003): Deficiencia auditiva. Evaluación, intervención y recursos psicológicos, CEPE, Madrid.

LEy ORGÁNICA DE EduCACIÓN, Título II: Equidad en la Educación (2/2006 del 3 de mayo).

Manolson, A. (1995): Hablando nos entendemos los dos, Hannen Centre, Canadá.

MONFORT M. (2006): La práctica de la comunicación Bimodal. Del signo a la palabra, Entha, Madrid.

Organización de las naciones Unidas para la Educación, la Ciencia y la CULTURA (2008), Ginebra: Suiza. Recuperado de http://www.ibe.unesco.org/fileadmin/user upload/Policy Dialogue/48th ICE/CONFINTED 48-3 Spanish.pdf.

SANCHIS, M. L. (2009): Modelos de orientación e intervención psicopedagógica, Colección Sapienta, Castellón de la Plana.

SERRANO BARQuÍN, C. (2008): Creatividad sensorial: sensopercepción y desarrollo humano, Pax, México.

BLUMA S. M. (1976): Guía Portage de Educación Preescolar, Ediciones TEA, Barcelona.

THORNE, K. (2008): Motivación y creatividad en clase, Ediciones Graó, Barcelona.

TORRES MONTREAL, S. (2001): Sistemas alternativos de comunicación. Manual de comunicación aumentativa i alternativa, Ediciones Aljibe, Málaga.

Von TetzChner, S. y H. MARTINSEN (1993): Introducción a la enseñanza de signos y al uso de ayudas técnicas para la comunicación, Antonio Machado Libros, Madrid. 
